\title{
Challenges and Opportunities to Harnessing the (Hematopoietic) Stem Cell Niche
}

\author{
Ji Sun Choi ${ }^{1}$ - Brendan A. C. Harley ${ }^{1,2}$
}

Published online: 29 January 2016

(C) Springer International Publishing AG 2016

\begin{abstract}
In our body, stem cells reside in a microenvironment termed the niche. While the exact composition and, therefore, the level of complexity of a stem cell niche can vary significantly tissue-to-tissue, the stem cell niche microenvironment is dynamic, typically containing spatial and temporal variations in both cellular, extracellular matrix, and biomolecular components. This complex flow of secreted or bound biomolecules, cytokines, extracellular matrix components, and cellular constituents all contributes to the regulation of stem cell fate specification events, making engineering approaches at the nanoscale and microscale of particular interest for creating an artificial niche environment in vitro. Recent advances in fabrication approaches have enabled biomedical researchers to capture and recreate the complexity of stem cell niche microenvironments in vitro. Such engineered platforms show promise as a means to enhance our understanding of the mechanisms underlying niche-mediated stem cell regulation as well as offer opportunities to precisely control stem cell expansion and differentiation events for clinical applications. While these principles generally apply to all adult stem cells and niches, in this review, we focus on recent developments in engineering synthetic niche microenvironments for one of the
\end{abstract}

This article is part of the Topical Collection on Stem Cells and Nanotechnologies

Brendan A. C. Harley

bharley@illinois.edu

1 Department of Chemical and Biomolecular Engineering, University of Illinois at Urbana-Champaign, 110 Roger Adams Laboratory, 600S. Mathews Ave., Urbana, IL 61801, USA

2 Carl R. Woese Institute for Genomic Biology, University of Illinois at Urbana-Champaign, 110 Roger Adams Laboratory, 600S. Mathews Ave., Urbana, IL 61801, USA best-characterized stem cell populations, hematopoietic stem cells (HSCs). Specifically, we highlight recent advances in platforms designed to facilitate the extrinsic control of HSC fate decisions.

Keywords Synthetic stem cell niche $\cdot$ Hematopoietic stem cells $\cdot$ Imaging

\section{Introduction}

\section{The Stem Cell Niche as a Biological Entity}

The genetic information required to direct stem cell fate specification events such as quiescence, self-renewal, or differentiation is contained within its DNA, but signals from its microenvironment - surrounding cells, the extracellular matrix (ECM), as well as ECM-bound or diffusible biomoleculestrigger these events $[1 \bullet \cdot, 2-4]$. These unique regionsniches - offer exciting opportunities to develop new bioengineering approaches that use nanoscale and microscale technologies to engineer cell fate $[1 \bullet \bullet, 5,6]$. An increasing number of adult stem cells and their corresponding niches have been identified in different tissues and organs, including skin, gut, bone marrow, and brain [7-13]. These stem cell niches are dynamic microenvironments that present combinations of cellular, ECM, and biomolecular cues $[1 \bullet \cdot, 11,14]$. Importantly, these extrinsic signals are in a continuous state of flux, resulting in complex network of secreted or bound biomolecules, cytokines, ECM, and cellular components with spatial and temporal variations $[1 \bullet \bullet, 3,14]$. The dynamic nature of these niches remains across a wide range of physiological stages, from development, homeostasis, and injury/stress responses through aging and senescence [9, 14-21]. These niches not only host a native stem cell population, but also 
provide vital extrinsic signals necessary for their survival, proliferation, differentiation, mobilization, and other functional activities $[3,10]$.

Hematopoiesis is the physiological process where a small number of hematopoietic stem cells (HSCs) continuously generate the body's full complement of blood and immune cells $[14,22,23]$. While HSC niches are found primarily in the bone marrow in adult vertebrates, during development HSCs and their niche transition between multiple anatomical locations $[9,15,16]$. Primitive hematopoiesis is first observed in the yolk sac [15]. Definite hematopoiesis then ensues in the aortagonad-mesonephros (AGM), placenta, fetal liver, and spleen and finally in the bone marrow, the primary site of hematopoiesis for adults $[9,16]$. During adult hematopoiesis, HSCs are found primarily in the bone marrow HSC niches, where various cellular components (e.g., osteolineage cells, vascular endothelial cells, neurons, macrophages), extracellular proteins (e.g., fibronectin, laminin, collagen, proteoglycans), and secreted or immobilized biomolecules and growth factors (e.g., stem cell factor (SCF), TPO, Ang-1, Flt3L, CXCL12, granulocytecolony stimulating factor (G-CSF), IL-3, IL-6, IL-11) comprise the functional microenvironments with local gradients in cellular and extracellular contents [2, 14, 19, 24-28]. Several discrete anatomical localizations within the marrow have been described for HSC niches (e.g., endosteal, perivascular, and, more specifically, sinusoidal and arteriolar niches) [19, 24, 27, $29,30]$. And, while it is unclear if they exist as completely separate or rather a series of overlapping microenvironments, these discrete sub-niches are believed to serve a differing role in HSC maintenance, differentiation, and mobilization [19, 24]. Recent reports suggest that aging also significantly alters the functional capacity of HSCs (e.g., diminished lymphoid potential) and that aging-induced changes in the HSC niche composition may contribute to the observed changes [20,21].

The stem cell niche is not just a physical space within a tissue or an organ but can be considered a machinery of its own, a highly organized and hierarchical biological entity that facilitates the maintenance and function of stem cells $[10,31$, 32]. Identifying the role played by biophysical and molecular features of the niche on HSC fate specification requires new

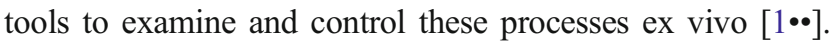
Bioengineering approaches may be particularly well suited to reduce the complexity of the niche to gain mechanistic insight regarding how the biophysical and molecular signal pathways shape HSC fate $[1 \bullet \bullet, 5,6,33]$. Through improved understanding of how the niche modulates complex stem cell behaviors, we can eventually gain the capacity to engineer stem cell fate decisions in vitro [6, 34-37]. In vivo studies that de-functionalize niches by removing cell or matrix constituents provide insight regarding niche-associated signaling molecules (e.g., SCF, CXCL12) [32, 38-40] and the potential role of mesenchymal stem cells (MSCs) as a niche cell to chaperone HSC fate specification [41]. However, given likely synergies and hierarchies between niche signals, knocking out elements of the larger network may identify central features but will not provide insight regarding design of an artificial marrow $[1 \bullet \cdot$. Mimicking the niche as a coordinated entity of action requires understanding HSC fate decisions in response to multiplexed cell, biophysical, and biomolecular signals $[1 \bullet \bullet, 10,34]$.

Stem cell engineering research is in the midst of a paradigm shift $[1 \bullet \cdot, 34,37,42]$. Historically, the study of niches has been restricted to in vivo efforts that defunctionalize an otherwise physiological niche or simplistic two-dimensional (2D) cultures [37, 42]. Both have limited capacity for examining hierarchies and synergies. Yet, as the prototype mammalian stem cell and given its long history of clinical use, we are armed with well-defined metrics to assess HSC stemness and are inspired by questions with direct clinical ramifications $[1 \bullet \cdot, 34,43]$.

\section{Technical Challenges for Engineering Synthetic Niches at the Nanoscale}

The regenerative potential of stem cells renders them attractive for several tissue engineering and regenerative medicine applications $[1 \bullet \bullet, 6,36,37,44 \bullet \cdot]$. The paucity of stem cells, however, requires our ability to precisely control their activities in vitro, particularly expansion and differentiation, in order to increase their potential clinical value $[1 \bullet \bullet, 44 \bullet \bullet, 45]$. Since multiple niche constituents have been shown previously to modulate stem cell behaviors, biomedical researchers in the field have been trying to recreate aspects of stem cell niche microenvironments in vitro to better understand nichemediated stem cell regulation as well as to better manipulate stem cell functions $[5,6,34,44 \cdot \bullet]$. While this approach has led to some creative means to expand or differentiate stem cells and new insights into stem cell maintenance, current technical limitations make it challenging to recapitulate the complexity of the niche architecture in vitro $[1 \bullet \bullet, 5,6,34$, $36,37,44 \cdot \bullet]$.

Beyond simply assembling the correct sequence of signals to induce a stem cell response, new methods are also needed to interrogate stem cell response to those signals in increasing detail. While not surprising, stem cells such as HSCs may exhibit a range of responses to a niche signal [17, 46, 47]. This heterogeneity will likely be magnified in multi-cue environments and must be defined $[1 \bullet \cdot$. Currently available functional assays or labeling techniques rarely allow in situ analysis of single, live stem cells, which may skew the characterization of stem cell responses to niche-mediated cues $[1 \bullet \bullet, 45$, 48]. This is particularly true for HSCs with known heterogeneities in their functional capacity, incompletely defined surface markers for isolation or identification, and lack of reliable in situ functional assays [17, 48, 49]. An opportunity therefore exists to miniaturize and parallelize engineered bone marrow 
constructs to compare the response of single HSCs to the ensemble average from HSC populations [1••, 45, 50]. New methods should gather accurate, quantitative information for single HSCs or small groups of HSCs within libraries of defined synthetic niches $[45,50]$. Such information will be critical to identify the minimum number of cells to resolve a fate decision and, consequently, the scale of a culture platform to induce a desired range of fates. Heterogeneity also offers a unique perspective for deciphering the relative importance of discrete cues, as reduced heterogeneity can often be correlated with heightened significance [51-53].

In this review, we highlight recent advances that attempt to overcome these technological hurdles: both assembling increasingly complex synthetic niches as well as tools to interrogate stem cell response. We discuss material-based approaches capable of modulating stem cell behaviors by providing extrinsic cues via the presentation of biomolecules or other extracellular factors at the cell-material interface down to nanoscale resolution, effectively mimicking cell-matrix and cell-cell interactions in the stem cell niche. We also briefly summarize new, innovative efforts to characterize stem cells at the molecular level with subcellular resolution. We focus on HSCs and their niches in the bone marrow, but the general principles should extend to other stem cells and niches as well.

\section{Niche-Mimicking Platforms for Modulating Stem Cell Behaviors}

Advances in fabrication approaches have enabled biomedical researchers to simultaneously integrate multiple aspects of the stem cell niche onto engineered cell-material interfaces $[1 \bullet \bullet, 6$, $33,35,44 \bullet \cdot]$. Here, we discuss recent developments, from simple 2D surfaces to sophisticated three-dimensional (3D) constructs. Matrix chemistries, mechanics, topographies, and dimensionality can all stimulate different features of stem cell fate decisions in vitro [36, 42], thereby enhancing our understanding of the niche-mediated stem cell regulation and, in some cases, establishing an effective means to control stem cell function in vitro (Fig. 1).

\section{Two-Dimensional Substrates and Liquid Cultures-Demonstrating Regulatory Concepts}

Two-dimensional material surfaces are relatively easy fabricate, and their material properties such as chemistries, topographies, and mechanics could be easily modified [35, 54]. It is therefore easy to decouple the effects of single or combined biomolecules (e.g., proteins, proteoglycans, growth factors), material properties (e.g., stiffness, elasticity, fiber strength), and structural details (e.g., topographical features, geometries, ligand spacing) on stem cell activities using these platforms $[1 \bullet \bullet, 6,33-36,42,44 \bullet \bullet$. Presentation of the biomolecules or material properties could be controlled to imitate stem cellmatrix and/or cell-cell interactions [6, 34, 36, 55•]. The obvious drawback is the lack of dimensionality that deviates from the physiological stem cell niche $[6,56]$.

As stem cell-material interactions are mediated by ligandreceptor binding that modulates downstream signal transduction events, several studies directly target receptor activation (e.g., integrins) by employing surfaces with nanoscale features [54]. Here, presentation of ligands or other cell adhesion molecules (e.g., full-length proteins, fragments, peptides) is controlled to produce surfaces with defined properties (e.g., ligand density, spatial orientation, spacing, chemistry) that could induce specific ligand-receptor interactions leading to desired stem cell activities [54, 57, 58••].

Matrix Cues Presentation of extracellular components or growth factors via surface immobilization of material substrates has revealed that HSC-matrix interactions significantly influence HSC adhesion [59], spreading [31], proliferation $[60,61]$, and differentiation [62]. HSCs show preferential adhesion to several extracellular matrix (ECM) proteins and proteoglycans $[28,59]$. When HSCs were grown on top of poly(ethylene-alt-maleic anhydride) (PEMA) substrates chemically modified with proteins (fibronectin, type I collagen fibrils, tropocollagen I) and proteoglycans (heparin sulfate, heparin, hyaluronic acid), HSCs adhered most strongly to fibronectin while moderately adhered to heparin sulfate, heparin, and collagen I fibrils and insignificantly adhered to tropocollagen I and hyaluronic acid [59]. HSC adhesion also affects its morphology, and HSCs spread out significantly more and appear increasingly irregular and less circular in shape when presented with increasing surface ligand density, as seen by HSC cultures on type I collagen-coated polyacrylamide gels [31]. Adhesion may affect proliferation activity as well. When whole bone marrow cells were cultured on plastic culture plates with physically adsorbed tropoelastin, the number of HSCs in the population significantly increased compared to the negative control, suggesting that changes in surface elasticity contributed to the observed HSC expansion [60]. Similarly, hematopoietic stem and progenitor cells (HSPCs) cultured on polyethylene glycol diacrylate (PEGDA) gel surfaces modified with SCF, IFN- $\gamma$, and Arg-Gly-Asp (RGD) showed considerably more expansion compared to unmodified counterparts, emphasizing the importance of sustained growth factor presentation in HSC growth [61]. In another study, magnetic microbeads functionalized with notch ligand delta-like ligand 4 (DLL4) via streptavidin-biotin conjugation facilitated HSC differentiation to T cell precursors when they were added to HSC co-cultures with stromal cells [62]. For ligand immobilization studies such as these, surface chemistry needs to be carefully considered as subtle changes may alter stem cell response $[44 \bullet \bullet]$. 
Fig. 1 Material fabrication approaches for stem cell nicheinspired extrinsic control of stem cell fate decisions in 2D and 3D synthetic niche microenvironments in vitro. Biomolecular presentation, mechanical properties, and structural details could be controlled to nanoscale resolution to replicate sophisticated features

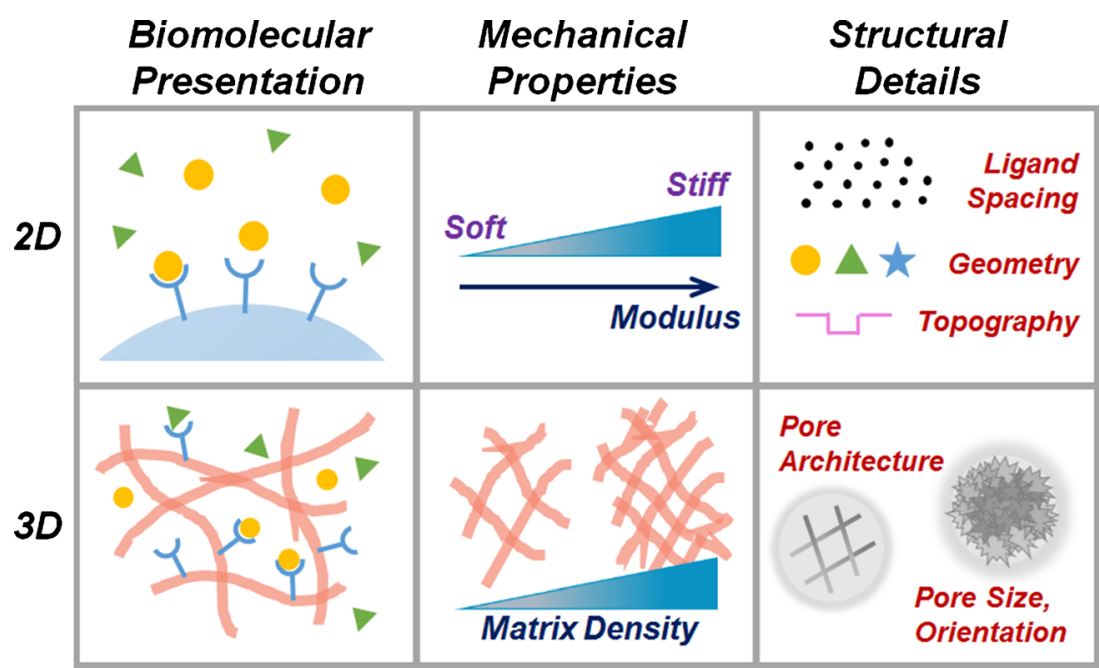

Mechanical Cues Changes in stiffness of 2D material surfaces have been linked to significant changes in HSC morphology [31] and motility [63]. When cultured on type I collagen-coated polyacrylamide gels with varying stiffness (0.71-196 kPa), HSCs spread out more and appeared less circular on stiffer substrates, exhibiting more irregular morphology [31]. On fibronectin-coated PEGDA substrates, HSCs adhered better and migrated faster to a chemotactic cue on stiffer $(>38 \mathrm{kPa})$ vs. softer $(\leq 20 \mathrm{kPa})$ substrates [63]. These studies suggest that HSCs are responsive to changes in mechanical cues, but more studies should be performed to assess how such cues lead to definitive functional changes in cultured HSCs (e.g., proliferation, differentiation).

Structural Signals Structural details such as topographical features or ligand spacing have been found to impact HSC quiescence [64, 65], adhesion [66], gene expression [66], and lipid raft clustering [67], rendering them an important design parameter for recreating HSC niches in vitro. When HSCs were cultured in ECM protein-functionalized polydimethylsiloxane (PDMS) microcavities $(15-80 \mu \mathrm{m}$ in diameter), more HSCs retained primitive HSC markers in smaller cavities, particularly when the cavities were coated with fibronectin [64] or heparin [65]. A study utilizing nanopatterned hydrogel substrates showed that HSC adhesion and gene expression are influenced by the lateral spacing of ligands [66]. Here, polyethylene glycol (PEG) substrates (Young's modulus of $6 \mathrm{MPa}$ ) decorated with ligand-functionalized gold nanoparticles spaced at varying lateral distance $(\sim 20-110 \mathrm{~nm})$ were prepared and HSCs were grown on top. Based on the ligand type, the critical interligand spacing that could support HSC adhesion differed (cRGD $<45 \mathrm{~nm}$, osteopontin $\sim 75 \mathrm{~nm}$, fibronectin domain III 7-10 containing RGD sequence $\sim 110 \mathrm{~nm}$ ), and such HSC-ligand interactions impacted the gene expression of thrombospondin-2 (THBS2), a glycoprotein involved in cell adhesion [68]. On similarly prepared cRGD- functionalized nanopatterned glass substrates, HSCs showed higher levels of adhesion and lipid raft clustering on substrates with smaller $(<32 \mathrm{~nm})$ lateral ligand spacing, where lipid raft clustering is believed to be key in activating several signal transduction events [67].

\section{Biomolecular Cues and Engineering Feedback Loops} Recent studies suggest that combining spatial and temporal aspects of small biomolecule presentation (e.g., cytokines, growth factors) may significantly enhance stem cell expansion by minimizing the impact of inhibitory signals, often secreted by differentiated cells in the culture population $[50,69,70]$. For instance, immobilized biomolecules mimicking membrane-bound isoforms could amplify desired output over soluble isoforms by providing sustained ligand-receptor interactions leading to prolonged receptor activation $[69,71]$. As such, immobilized SCF (a key cytokine known to be involved in HSC survival and maintenance [72]) was shown to enhance adhesion and proliferation activity of primary HSCs and hematopoietic cell lines compared to soluble SCF [61, 71, 73-75]. Additionally, temporal regulation of biomolecular presentation (e.g., availability, diffusive transport) has been found to be important in modulating HSC activities [50, 70, 76-78]. Single-cell studies have demonstrated that cytokine administration (M-CSF or G-CSF) could directly instruct lineage choice in hematopoietic progenitor cell populations instead of simply expanding already committed subpopulations [76]. Feedback loop studies have allowed characterization of HSC culture kinetics in 2D liquid cultures, where observed heterogeneities in proliferation and differentiation rates were linked to variations in intercellular signaling (autocrine or paracrine) $[70,77]$. By constructing a mathematical feedback model and altering parameters for the HSC culture systems (e.g., media exchange frequency, initial cell seeding density, fed-batch culture volume dilution rate, surface ligand 
immobilization), culture conditions could be optimized to maximize HSC expansion [70, 77-79].

Other Approaches A recent study employing MSC-secreted extracellular matrix (ECM) as a surface for HSC cultures demonstrates a new way of mimicking the stem cell niche in vitro [80]. Here, the researchers did not create the material interface themselves but employed niche cells to secrete matrix materials. MSC-secreted ECM matrix was produced by culturing MSCs with or without macromolecular crowding (MMC) for 10 days. MMC refers to polymer particles added to the cell culture media to occupy some of the culture space and stimulate growth factor secretion [81]. ECM matrix produced from MSCs cultured with MMC showed higher ECM content, higher overall glycosaminoglycan (GAG) content, and higher concentrations of certain growth factor (FGF-2, VEGF, HGF) released back to the media. When HSCs were cultured on top of this matrix for 7 days, there was a significant increase in HSC expansion compared to those cultured on the matrix produced without MMC. This study suggests that niche cells could be effectively manipulated to produce surfaces with niche-mimicking details.

\section{Three-Dimensional Biomaterial Niches—Exploring New Frontier for Control}

Like the niches that they attempt to replicate, approaches to engineer stem cell fate specification events increasingly present complex combinations of extrinsic cues. The added dimensionality in 3D biomaterials creates sites for cell-matrix interactions, covalently bound morphogens, and diffusive transport of soluble molecules [35, 37]. This allows better recapitulation of autocrine or paracrine signaling, local gradients of nutrients and oxygen, diffusion rates of biomolecules, and structural and adhesive support in vitro [6]. Thus, 3D constructs offer the potential to recreate a dynamic microenvironment that is more similar to the physiological stem cell niche $[82,83]$.

Similar to 2D material surfaces, 3D biomaterials can be chemically modified to include cell adhesion (e.g., RGD), degradation moieties (e.g., MMP degradable sites), and other biomolecules (e.g., growth factors, cytokines, extracellular components) to modulate stem cell behaviors $[84,85]$. Their material properties can also be tuned to exhibit varying mechanics and architecture [35, 84]. While 3D constructs may be limited to certain fabrication approaches due to cell encapsulation needs [86, 87], recent advances facilitate the formation of 3D microenvironments with high spatial resolution, even down to nanometer-scale features [88, 89]. Some of them allow temporal polymerization or degradation of the support structure as well to dynamically mimic aspects of stem cellcell and stem cell-matrix interactions within the stem cell niche $[85,90,91]$.
Here, we describe several recent developments in 3D constructs for recreating HSC bone marrow niche, which have been critically linked to HSC activities including adhesion [92], proliferation [61, 73, 92-94], and engraftment [92]. A wide range of synthetic 3D HSC niches utilize co-culture with other cells, typically a variation of mesenchymal or stromal cells, in order to promote increased cell survivability and growth support $[1 \bullet \bullet$.

Biomolecular Signals Like 2D substrates, 3D biomaterial constructs offer opportunities to present biomolecular signals in both matrix-bound and soluble form and additionally alter biotransport of soluble factors within the matrix [85]. In a recent study, the effect of bound vs. soluble SCF presentation in HSC maintenance was examined by culturing HSCs in methacrylamide-functionalized gelatin (GelMA) hydrogels with or without covalently attached SCF for up to 7 days [73]. SCF is a cytokine critical for HSC quiescence and activation [40], and gelatin, which is the denatured form of collagen, naturally promotes cell adhesion and enzymatic degradation [95]. GelMA gels with immobilized SCF could maintain a higher percentage of primitive HSCs with low overall proliferation, whereas GelMA gels containing only soluble SCF saw higher overall proliferation but a lower percentage of primitive HSCs due to increased differentiation activity [73]. In other studies, HSPCs were cultured in PEGDA hydrogels or scaffolds functionalized with RGD motifs $[61,93]$. PEGDA hydrogels also contained MMP-degradable sites and could support long-term cultures (up to 5 weeks) of HSPCs [61]. PEGDA scaffolds were fabricated by salt leaching technology and could enhance HSC expansion when HSCs were co-cultured with bone marrow-derived MSCs [93].

Another aspect of biomolecular supplementation approaches for mimicking the stem cell niche is taking advantage of non-covalent interactions to fine-tune stem cell response via growth factor sequestration [96, 97]. Recent efforts for MSC expansion and musculoskeletal repair leveraging proteoglycan-inspired transient sequestration and molecular imprinting technologies demonstrate the importance of sequestration strategies, offering exciting opportunities to further control and refine stem cell response [98]. Here, chargebased or conformation-based biomolecule interactions within constructs could sequester exogenously added or endogenously produced biomolecular signals to enhance responses such as proliferation and fate specification [99-101]. As such, integrating charged proteoglycans such as heparin or chondroitin sulfate into $3 \mathrm{D}$ constructs has been shown to strengthen signaling pathways $[98,99]$ and amplify gene delivery $[101]$ to boost MSC response.

Microstructural Signals The effect of dimensionality on HSC cultures has been explored by comparing bone-derived 
3D scaffolds and traditional 2D tissue culture plates in supporting HSC expansion [94]. Both the scaffolds and tissue culture plates were pre-seeded with MSCs to simulate an osteoblastic environment. Here, 3D scaffolds performed significantly better than 2D substrates in fostering HSC expansion, probably due to the $3 \mathrm{D}$ environment enhancing MSCmediated HSC support and possibly overall support for cell growth and proliferation. In a separate study, HSCs were cultured in polycaprolactone (PCL), poly(lactide-co-glycolide) (PLGA), fibrin, or collagen scaffolds for expansion [92]. Fibrin scaffolds were found to best support HSC expansion and engraftment, particularly when HSCs were co-cultured with MSCs from umbilical cord. In contrast, PCL scaffolds showed insignificant expansion with low levels of observed cell adhesion, likely due to differences in scaffold architecture and mechanical properties such as pore or fiber geometries, size, orientation, and stiffness.

Microphysiological Systems Microfluidic platforms have been increasingly used to make in vitro culture systems with more sophisticated control over spatial and temporal aspects of the culture microenvironments [102-104]. Several microfluidic-based approaches have been developed for HSCs, aiding with the incorporation of cellular and extracellular components and the administration of growth factors and cytokines to create a functional artificial stem cell niche $[1 \bullet \bullet$. For instance, a two-input microfluidic mixer allows easy fabrication of 3D hydrogels with opposing gradients of cells, matrix, and other biomolecules [73, 105]. As a proof of concept, opposing gradients of HSCs and osteoblasts [105] and a gradient of GelMA hydrogels with covalently crosslinked SCF [73] were successfully created in microliter-scale $(\sim 180 \mu \mathrm{L})$ cultures. Further work is underway in order to incorporate additional factors to tease out how local gradients impact HSC activities. In a different study, a microfluidicbased microarray of nanoliter-scale chambers was fabricated for high-throughput analysis [106]. Although this example did not produce 3D microenvironments, thousands of chambers of HSC cultures were automatically replenished with culture media containing desired amounts of growth factors. Such temporal control combined with live-cell time-lapse imaging revealed that while SCF concentration is not important during first division, SCF is required for survival afterwards. These reports illustrate that microfluidics is a promising tool for reconstructing complex, dynamic stem cell niche microenvironments with high degrees of spatial and temporal control.

\section{Techniques to Non-Invasively Monitor Stem Cell Response}

An important aspect of developing niche-mediated stem cell regulation schemes is the development of new analytical approaches to quantify HSC response to unique combinations of niche signals with high fidelity $[1 \bullet \bullet]$. As engineered niche signals are increasingly complex and dynamic in nature, it is essential to profile stem cell responses with similar rigor. While not surprising, HSCs may exhibit a range of responses to a niche signal, and this heterogeneity will likely be magnified in multi-cue environments and must be defined [46, 107]. Ideally, stem cell functional metrics should provide convenient yet accurate quantification of subtle changes in individual, live stem cells in situ, dynamically assessing single cells with minimal sample manipulation to avoid disturbing the cells or culture conditions $[1 \bullet \cdot$. Current functional measures of HSCs include transplantation assays, clonal expansion assays (e.g., colony-forming unit (CFU) assay, cobblestone area-forming cell (CAFC) assay, long-term culture-initiating cell (LTC-IC) assay), and fluorescent labeling based on an incompletely defined set of surface markers, which are endpoint analyses that require long incubation time or extensive staining $[48,108]$. An opportunity therefore exists to miniaturize and parallelize engineered bone marrow constructs to compare the response of single HSCs to the ensemble averages from HSC populations $[1 \bullet \bullet, 45,107]$. However, while engineering approaches offer the potential to systematically vary the local microenvironment surrounding HSCs in order to dissect the importance of individual or small groups of extrinsic signals on cell fate, recapitulating the entirety of the complex interactions which take place in vivo remains an ongoing technical challenge. Further, such increasingly complex cultures also introduce new areas of consideration with significant opportunity for innovation. Namely, while surface antigen expression profiles remain the current gold standard for identifying stem cell phenotypes, label-free approaches probing cell-specific molecular or adhesive information would facilitate the characterization of dynamic HSC fate specification events.

Chemical Fingerprint In a recent report, our group has demonstrated a label-free approach to quantify HSC functional capacity using the evolving chemical spectra of the cell membrane [109]. In this study, fixed primary hematopoietic cell populations (HSPCs, common lymphoid progenitors (CLPs), and mature B cells) down the HSC differentiation hierarchy from old (10 months old) vs. young (2-4 months old) mice were probed with time-of-flight secondary ion mass spectrometry (TOFSIMS), and the resulting mass spectral datasets were subject to multi-variate analysis (MVA). MVA of TOF-SIMS data for individual hematopoietic cells could be used to identify cell type as well as cells from old vs. young mice with high sensitivity and selectivity with low identification errors: 3,8 , and $11 \%$ error for $\mathrm{B}$ cells, CLPs, and HSCs, respectively, for cell type identification and 19, 26, and $11 \%$ error for B cells, CLPs, and HSCs, respectively, for identifying cells from old vs. young mice. More recently, we used Raman spectroscopy to probe fixed primary 
hematopoietic cells (long-term HSCs (LT-HSC), short-term HSCs (ST-HSC), mature B cells, and granulocytes) seeded on hydrogel substrates to collect their molecular information in situ [110]. MVA of the resulting Raman spectra of individual cells could successfully identify cell type with low identification errors even when the cells were seeded on different hydrogels (3, 4,0 , and $2 \%$ error for LT-HSCs, ST-HSCs, granulocytes, and B cells, respectively, for overall cell identification and 4, 5, 10, and $3 \%$ error for LT-HSCs, ST-HSCs, granulocytes, and B cells, respectively, for cell identification when cells were seeded on different hydrogel substrates). These results demonstrate the feasibility of using single-cell molecular information as a "chemical fingerprint" for in situ cell identification. Ongoing work is employing analytical approaches to probe live cells in situ.

Adhesive Signature Niche cells comprise a significant fraction of the stem cell niche, and single-cell analytical approaches that could monitor and rapidly differentiate HSCs from niches cells in multi-cellular cultures are highly desirable. The capacity to profile the functional status (e.g., division, differentiation, migratory patterns) of a single stem cell opens opportunities to define the life history of that stem cell, cataloging not only HSC phenotype but also the trajectory of cell-cell interactions that take place in both HSC monocultures as well as more complex cultures that combine HSCs with niche cells $[45,107]$. To this end, label-free time-lapse tracing of HSC fate decisions in response to multiplexed cell and biophysical signals offers an exciting frontier for biomedical advances as they do not require significant manipulation of the stem cells before or during the experiment $[1 \bullet \bullet]$. In our recent reports, we have demonstrated the feasibility of applying photonic crystal enhanced microscopy (PCEM) as a label-free, in situ analysis approach for probing how individual cells adhere to ligand-coated surfaces (e.g., cell placement, intracellular focal adhesion complex distributions, migratory speed, and projection) [111-113]. PCEM is a modified brightfield microscope setup with a low-intensity non-coherent light source, utilizing ligand-modified photonic crystal surfaces as the biosensor interface. The photonic crystal surfaces have periodic nanograting structures that produce an evanescence field on top when light at a specific wavelength is shone through. When cells are grown on top of the biosensor and cellular materials come in direct contact with the surface, wavelength shifts occur in the light passing through, which can be collected to quantify changes in cell intrinsic properties (e.g., dielectric permittivity) with submicron spatial resolution to enable rapid adhesive phenotyping of individual cells without the use of exogenous labels. Proof-of-concept studies with dental epithelial stem cells showed the capacity of PCEM to dynamically track and categorize cells during chemotaxis, suggesting that PCEM can be non-invasively applied to classify live stem cells.

\section{Conclusions and Future Opportunities}

Recent advances in fabrication approaches enable microscale and nanoscale details of the complex stem cell niche microenvironments to be replicated in vitro to build sophisticated 2D and 3D biomaterials serving as synthetic niches. Such synthetic niche systems allow mechanisms of niche action on stem cell regulation to be uncovered and elucidated while also providing the means to precisely control stem cell activities to maximize their clinical value. Additionally, advances in label-free approaches increasingly permit quantification of single stem cell fate decisions to unique combinations of extrinsic signals with high fidelity in order to characterize the trajectory of cell-cell and cell-matrix interactions in stem cell monocultures as well as multi-cellular cultures with stem and niche cells. In time, these methods would lead to dynamic time-lapse tracing of single stem cell fate decisions in situ, facilitating characterization and tuning of stem cell response to multiplexed signals. Going forward, development of integrated material platforms that capture spatial and temporal variations of the stem cell niche with builtin analysis modules to detect and categorize single stem cell activities in situ in a label-free manner would greatly enhance the extrinsic control of stem cells. For this, leveraging nanotechnological advances would be crucial to harness the full potential of the stem cell niche.

Acknowledgments This material is based upon work supported by the National Science Foundation under Grant No. 1254738. Research reported in this publication was also supported by NIH R01 DK099528 and R21 EB018481. The content is solely the responsibility of the authors and does not necessarily represent the official views of the NIH. The authors confirm that there are no known conflicts of interest associated with this publication and there has been no significant financial support for this work that could have influenced its outcome.

\section{Compliance with Ethical Standards}

Conflict of Interest Ji Sun Choi and Brendan A. C. Harley declare that they have no conflict of interest.

Human and Animal Rights and Informed Consent This article does not contain any studies with human or animal subjects performed by any of the authors

\section{References}

Papers of particular interest, published recently, have been highlighted as:

- Of importance

•- Of major importance

1.• Choi JS, Mahadik BP, Harley BAC. Engineering the hematopoietic stem cell niche: frontiers in biomaterial science. Biotechnol J. 
2015;10(10):1529-45. doi:10.1002/biot.201400758. This review provides a detailed overview of strategies for engineering the hematopoietic stem cell niche in vitro.

2. Hines M, Nielsen L, Cooper-White J. The hematopoietic stem cell niche: what are we trying to replicate? J Chem Technol Biot. 2008;83(4):421-43.

3. Jones DL, Wagers AJ. No place like home: anatomy and function of the stem cell niche. Nat Rev Mol Cell Bio. 2008;9(1):11-21. doi:10.1038/Nrm2319.

4. Smith JN, Calvi LM. Concise review: current concepts in bone marrow microenvironmental regulation of hematopoietic stem and progenitor cells. Stem Cells. 2013;31(6):1044-50. doi:10.1002/ stem.1370.

5. Lee-Thedieck C, Spatz JP. Artificial niches: biomimetic materials for hematopoietic stem cell culture. Macromol Rapid Commun. 2012;33(17):1432-8. doi:10.1002/marc.201200219.

6. Lee-Thedieck C, Spatz JP. Biophysical regulation of hematopoietic stem cells. Biomater Sci. 2014;2(11):1548-61. doi:10.1039/ c4bm00128a.

7. Young HE, Black AC. Adult stem cells. Anat Rec A: Discov Mol Cell Evol Biol. 2004;276A(1):75-102. doi:10.1002/ar.a.10134.

8. Nombela-Arrieta C, Pivarnik G, Winkel B, Canty KJ, Harley B, Mahoney JE et al. Quantitative imaging of haematopoietic stem and progenitor cell localization and hypoxic status in the bone marrow microenvironment. Nat Cell Biol. 2013;15(5):533-43. doi:10.1038/ncb2730 http://www.nature.com/ncb/journal/v15/n5/ abs/ncb2730.html\#supplementary-information.

9. Purton LE, Scadden DT. The hematopoietic stem cell niche. Cambridge: StemBook; 2008.

10. Scadden DT. The stem-cell niche as an entity of action. Nature. 2006;441(7097):1075-9. doi:10.1038/nature04957.

11. Wilson A, Trumpp A. Bone-marrow haematopoietic-stem-cell niches. Nat Rev Immunol. 2006;6(2):93-106. doi:10.1038/ Nri1779.

12. Kopp HG, Avecilla ST, Hooper AT, Rafii S. The bone marrow vascular niche: home of HSC differentiation and mobilization. Physiology. 2005;20:349-56. doi:10.1152/physiol.00025.2005.

13. Adams GB, Scadden DT. The hematopoietic stem cell in its place. Nat Immunol. 2006;7(4):333-7.

14. Mendelson A, Frenette PS. Hematopoietic stem cell niche maintenance during homeostasis and regeneration. Nat Med. 2014;20(8):833-46. doi:10.1038/nm.3647.

15. McGrath KE, Palis J. Hematopoiesis in the yolk sac: more than meets the eye. Exp Hematol. 2005;33(9):1021-8. doi:10.1016/j. exphem.2005.06.012.

16. Wang LD, Wagers AJ. Dynamic niches in the origination and differentiation of haematopoietic stem cells. Nat Rev Mol Cell Biol. 2011;12(10):643-55. doi:10.1038/nrm3184.

17. Babovic S, Eaves CJ. Hierarchical organization of fetal and adult hematopoietic stem cells. Exp Cell Res. 2014;329(2):185-91. doi: 10.1016/j.yexcr.2014.08.005.

18. Krause DS, Scadden DT, Preffer FI. The hematopoietic stem cell niche-home for friend and foe? Cytometry B Clin Cytom. 2013;84B(1):7-20. doi:10.1002/cyto.b.21066.

19. Morrison SJ, Scadden DT. The bone marrow niche for haematopoietic stem cells. Nature. 2014;505(7483):327-34. doi: 10.1038/nature12984.

20. McKerrell T, Vassiliou GS. Aging as a driver of leukemogenesis. Sci Trans Med. 2015;7(306):306fs38-fs38. doi:10.1126/ scitranslmed.aac4428.

21. Geiger H, de Haan G, Florian MC. The ageing haematopoietic stem cell compartment. Nat Rev Immunol. 2013;13(5):376-89. doi:10.1038/nri3433.

22. Kondo M, Wagers AJ, Manz MG, Prohaska SS, Scherer DC, Beilhack GF, et al. Biology of hematopoietic stem cells and progenitors: implications for clinical application. Annu Rev of
Immunol. 2003;21(1):759-806. doi:10.1146/annurev.immunol. 21.120601.141007.

23. Mosaad YM. Hematopoietic stem cells: an overview. Transfus Apher Sci. 2014;51(3):68-82. doi:10.1016/j.transci.2014.10.016.

24. Ehninger A, Trumpp A. The bone marrow stem cell niche grows up: mesenchymal stem cells and macrophages move in. J Exp Med. 2011;208(3):421-8. doi:10.1084/Jem.20110132.

25. Zon LI. Intrinsic and extrinsic control of haematopoietic stem-cell self-renewal. Nature. 2008;453(7193):306-13.

26. Anthony BA, Link DC. Regulation of hematopoietic stem cells by bone marrow stromal cells. Trends Immunol. 2014;35(1):32-7.

27. Silberstein LE, Lin CP. A new image of the hematopoietic stem cell vascular niche. Cell Stem Cell. 2013;13(5):514-6. doi:10. 1016/j.stem.2013.10.012.

28. Klamer S, Voermans C. The role of novel and known extracellular matrix and adhesion molecules in the homeostatic and regenerative bone marrow microenvironment. Cell Adhes Migr. 2014;8(6): 563-77. doi:10.4161/19336918.2014.968501.

29. Kunisaki Y, Bruns I, Scheiermann C, Ahmed J, Pinho S, Zhang D, et al. Arteriolar niches maintain haematopoietic stem cell quiescence. Nature. 2013;502(7473):637-43. doi:10.1038/ nature 12612 .

30. Acar M, Kocherlakota KS, Murphy MM, Peyer JG, Oguro H, Inra $\mathrm{CN}$ et al. Deep imaging of bone marrow shows non-dividing stem cells are mainly perisinusoidal. Nature. 2015;526(7571):126-30. doi:10.1038/nature15250 http://www.nature.com/nature/journal/ v526/n7571/abs/nature15250.html\#supplementary-information.

31. Choi JS, Harley BAC. The combined influence of substrate elasticity and ligand density on the viability and biophysical properties of hematopoietic stem and progenitor cells. Biomaterials. 2012;33(18):4460-8. doi:10.1016/j.biomaterials.2012.03.010.

32. Driessen RL, Johnston HM, Nilsson SK. Membrane-bound stem cell factor is a key regulator in the initial lodgment of stem cells within the endosteal marrow region. Exp Hematol. 2003;31(12): 1284-91.

33. Lutolf MP, Hubbell JA. Synthetic biomaterials as instructive extracellular microenvironments for morphogenesis in tissue engineering. Nat Biotechnol. 2005;23(1):47-55.

34. Peerani R, Zandstra PW. Enabling stem cell therapies through synthetic stem cell-niche engineering. J Clin Invest. 2010;120(1):60-70. doi:10.1172/Jci41158.

35. Higuchi A, Ling Q-D, Chang Y, Hsu S-T, Umezawa A. Physical cues of biomaterials guide stem cell differentiation fate. Chem Rev. 2013;113(5):3297-328. doi:10.1021/cr300426x.

36. Nava MM, Raimondi MT, Pietrabissa R. Controlling self-renewal and differentiation of stem cells via mechanical cues. J Biomed Biotechnol. 2012;2012:12. doi:10.1155/2012/797410.

37. Vunjak-Novakovic G, Scadden DT. Biomimetic platforms for human stem cell research. Cell Stem Cell. 2011;8(3):252-61. doi:10. 1016/j.stem.2011.02.014.

38. Kiel MJ, Morrison SJ. Maintaining hematopoietic stem cells in the vascular niche. Immunity. 2006;25(6):862-4.

39. Sugiyama T, Kohara H, Noda M, Nagasawa T. Maintenance of the hematopoietic stem cell pool by CXCL12-CXCR4 chemokine signaling in bone marrow stromal cell niches. Immunity. 2006;25(6):977-88.

40. Ding L, Saunders TL, Enikolopov G, Morrison SJ. Endothelial and perivascular cells maintain haematopoietic stem cells. Nature. 2012;481(7382):457-62. doi:10.1038/nature10783.

41. Mendez-Ferrer S, Michurina TV, Ferraro F, Mazloom AR, Macarthur BD, Lira SA, et al. Mesenchymal and haematopoietic stem cells form a unique bone marrow niche. Nature. 2010;466(7308):829-34. doi:10.1038/nature09262.

42. Burdick JA, Vunjak-Novakovic G. Engineered microenvironments for controlled stem cell differentiation. Tissue Eng A. 2008;15(2):205-19. doi:10.1089/ten.tea.2008.0131. 
43. Challen GA, Boles N, Lin KK, Goodell MA. Mouse hematopoietic stem cell identification and analysis. Cytometry A J Int Soc Anal Cytol. 2009;75(1):14-24. doi:10.1002/cyto.a.20674.

44.• Celiz AD, Smith JGW, Langer R, Anderson DG, Winkler DA, Barrett DA, et al. Materials for stem cell factories of the future. Nat Mater. 2014;13(6):570-9. doi:10.1038/nmat3972. This review provides a detailed overview of materials approaches for utilizing pluripotent stem cells for clinical applications. General principles discussed here could be extended to other types of stem cells as well.

45. Kokkaliaris KD, Loeffler D, Schroeder T. Advances in tracking hematopoiesis at the single-cell level. Curr Opin Hematol. 2012;19(4):243-9. doi:10.1097/MOH.0b013e32835421de.

46. Copley Michael R, Beer Philip A, Eaves CJ. Hematopoietic stem cell heterogeneity takes center stage. Cell Stem Cell. 2012;10(6): 690-7. doi:10.1016/j.stem.2012.05.006.

47. Schroeder T. Tracking hematopoiesis at the single cell level. In: Kanz L, Weisel KC, Orlic D, Fibbe WE, editors. Hematopoietic stem cells V. Annals of the New York Academy of Sciences. 2005:201-9.

48. Purton LE, Scadden DT. Limiting factors in murine hematopoietic stem cell assays. Cell Stem Cell. 2007;1(3):263-70. doi:10.1016/j. stem.2007.08.016.

49. Weissman IL, Shizuru JA. The origins of the identification and isolation of hematopoietic stem cells, and their capability to induce donor-specific transplantation tolerance and treat autoimmune diseases. Blood. 2008;112(9):3543-53. doi:10.1182/blood-2008-08078220

50. Endele M, Etzrodt M, Schroeder T. Instruction of hematopoietic lineage choice by cytokine signaling. Exp Cell Res. 2014;329(2): 207-13. doi:10.1016/j.yexcr.2014.07.011

51. Shi Q, Qin L, Wei W, Geng F, Fan R, Shin YS, et al. Single-cell proteomic chip for profiling intracellular signaling pathways in single tumor cells. Proc Natl Acad Sci U S A. 2012;109(2):41924. doi:10.1073/pnas.1110865109.

52. Silva J, Smith A. Capturing pluripotency. Cell. 2008;132(4):532-6.

53. Huang S. Non-genetic heterogeneity of cells in development: more than just noise. Development. 2009;136(23):3853-62. doi: 10.1242/dev.035139.

54. Prowse ABJ, Chong F, Gray PP, Munro TP. Stem cell integrins: implications for ex-vivo culture and cellular therapies. Stem Cell Res. 2011;6(1):1-12. doi:10.1016/j.scr.2010.09.005.

55. Watt FM, Huck WTS. Role of the extracellular matrix in regulating stem cell fate. Nat Rev Mol Cell Biol. 2013;14(8):467-73. doi: $10.1038 / \mathrm{nrm} 3620$. This review extensively confers the role of stem cell-matrix interactions in stem cell fate regulation.

56. Baker BM, Chen CS. Deconstructing the third dimension-how 3D culture microenvironments alter cellular cues. J Cell Sci. 2012;125(13):3015-24. doi:10.1242/jcs.079509.

57. Shekaran A, Garcia AJ. Nanoscale engineering of extracellular matrix-mimetic bioadhesive surfaces and implants for tissue engineering. Biochim Biophys Acta Gen Subj. 2011;1810(3):350-60. doi:10.1016/j.bbagen.2010.04.006.

$58 . \cdots$ Dalby MJ, Gadegaard N, Oreffo ROC. Harnessing nanotopography and integrin-matrix interactions to influence stem cell fate. Nat Mater. 2014;13(6):558-69. doi:10.1038/ nmat3980. This review provides a thorough overview of stem cell fate regulation via integrin-mediated cell adhesion to nanotopographical features on materials surfaces.

59. Franke K, Pompe T, Bornhauser M, Werner C. Engineered matrix coatings to modulate the adhesion of CD133(+) human hematopoietic progenitor cells. Biomaterials. 2007;28(5):836-43. doi:10. 1016/j.biomaterials.2006.09.031.

60. Holst J, Watson S, Lord MS, Eamegdool SS, Bax DV, NivisonSmith LB, et al. Substrate elasticity provides mechanical signals for the expansion of hemopoietic stem and progenitor cells. Nat Biotechnol. 2010;28(10):1123-8.
61. Cuchiara ML, Coşkun S, Banda OA, Horter KL, Hirschi KK, West JL. Bioactive poly(ethylene glycol) hydrogels to recapitulate the HSC niche and facilitate HSC expansion in culture. Biotechnol Bioeng. 2015. doi:10.1002/bit.25848.

62. Taqvi S, Dixit L, Roy K. Biomaterial-based notch signaling for the differentiation of hematopoietic stem cells into T cells. J Biomed Mater Res A. 2006;79A(3):689-97. doi:10.1002/jbm.a.30916.

63. Lee-Thedieck C, Rauch N, Fiammengo R, Klein G, Spatz JP. Impact of substrate elasticity on human hematopoietic stem and progenitor cell adhesion and motility. J Cell Sci. 2012;125(16): 3765-75. doi:10.1242/Jcs.095596.

64. Kurth I, Franke K, Pompe T, Bornhauser M, Werner C. Hematopoietic stem and progenitor cells in adhesive microcavities. Integr Biol. 2009;1(5-6):427-34. doi:10.1039/ b903711j.

65. Kurth I, Franke K, Pompe T, Bornhauser M, Werner C. Extracellular matrix functionalized microcavities to control hematopoietic stem and progenitor cell fate. Macromol Biosci. 2011;11(6):739-47. doi:10.1002/mabi.201000432.

66. Muth CA, Steinl C, Klein G, Lee-Thedieck C. Regulation of hematopoietic stem cell behavior by the nanostructured presentation of extracellular matrix components. Plos One. 2013;8(2). doi: 10. 1371/journal.pone.0054778.

67. Altrock E, Muth CA, Klein G, Spatz JP, Lee-Thedieck C. The significance of integrin ligand nanopatterning on lipid raft clustering in hematopoietic stem cells. Biomaterials. 2012;33(11):310718. doi:10.1016/j.biomaterials.2012.01.002.

68. Calabro NE, Kristofik NJ, Kyriakides TR. Thrombospondin-2 and extracellular matrix assembly. Biochim Biophys Acta Gen Subj. 2014;1840(8):2396-402. doi:10.1016/j.bbagen.2014.01.013.

69. Zandstra PW, Lauffenburger DA, Eaves CJ. A ligand-receptor signaling threshold model of stem cell differentiation control: a biologically conserved mechanism applicable to hematopoiesis. Blood. 2000;96(4):1215-22.

70. Csaszar E, Kirouac DC, Yu M, Wang W, Qiao W, Cooke MP, et al. Rapid expansion of human hematopoietic stem cells by automated control of inhibitory feedback signaling. Cell Stem Cell. 2012;10(2):218-29. doi:10.1016/j.stem.2012.01.003.

71. Doran MR, Markway BD, Aird IA, Rowlands AS, George PA, Nielsen LK, et al. Surface-bound stem cell factor and the promotion of hematopoietic cell expansion. Biomaterials. 2009;30(25): 4047-52. doi:10.1016/j.biomaterials.2009.04.043.

72. Kishimoto S, Nakamura S, Hattori H, Oonuma F, Kanatani Y, Tanaka Y, et al. Human stem cell factor (SCF) is a heparin-binding cytokine. J Biochem. 2009;145(3):275-8. doi:10.1093/jb/mvn169.

73. Mahadik BP, Haba SP, Skertich LJ, Harley BAC. The use of covalently immobilized stem cell factor to selectively affect hematopoietic stem cell activity within a gelatin hydrogel. Biomaterials. 2015;67: 297-307. doi:10.1016/j.biomaterials.2015.07.042.

74. Cuchiara ML, Horter KL, Banda OA, West JL. Covalent immobilization of stem cell factor and stromal derived factor 1alpha for in vitro culture of hematopoietic progenitor cells. Acta Biomater. 2013. doi:10.1016/j.actbio.2013.08.012.

75. Kishimoto S, Nakamura S, Hattori H, Oonuma F, Kanatani Y, Tanaka Y, et al. Cytokine-immobilized microparticle-coated plates for culturing hematopoietic progenitor cells. J Control Release Off J Control Release Soc. 2009;133(3):185-90. doi:10.1016/j. jconrel.2008.10.005.

76. Rieger MA, Hoppe PS, Smejkal BM, Eitelhuber AC, Schroeder T. Hematopoietic cytokines can instruct lineage choice. Science. 2009;325(5937):217-8. doi:10.1126/science.1171461.

77. Kirouac DC, Madlambayan GJ, Yu M, Sykes EA, Ito C, Zandstra PW. Cell-cell interaction networks regulate blood stem and progenitor cell fate. Molecular Systems Biology. 2009;5. doi:10. 1038/msb.2009.49. 
78. Csaszar E, Chen K, Caldwell J, Chan W, Zandstra PW. Real-time monitoring and control of soluble signaling factors enables enhanced progenitor cell outputs from human cord blood stem cell cultures. Biotechnol Bioeng. 2014;111(6):1258-64. doi:10.1002/bit.25163.

79. Csaszar E, Wang W, Usenko T, Qiao W, Delaney C, Bernstein ID, et al. Blood stem cell fate regulation by Delta-1-mediated rewiring of IL-6 paracrine signaling. Blood. 2014;123(5):650-8. doi:10. 1182/blood-2013-08-520445.

80. Prewitz MC, Stißel A, Friedrichs J, Träber N, Vogler S, Bornhäuser M, et al. Extracellular matrix deposition of bone marrow stroma enhanced by macromolecular crowding. Biomaterials. 2015;73:60-9. doi:10.1016/j.biomaterials.2015.09.014.

81. Zeiger AS, Loe FC, Li R, Raghunath M, Van Vliet KJ. Macromolecular crowding directs extracellular matrix organization and mesenchymal stem cell behavior. Plos ONE. 2012;7(5): e37904. doi:10.1371/journal.pone.0037904.

82. F-W Greiner J, Kaltschmidt B, Kaltschmidt C, Widera D. Going 3D - cell culture approaches for stem cell research and therapy. Curr Tissue Eng. 2013;2(1):8-19.

83. Ranga A, Gobaa S, Okawa Y, Mosiewicz K, Negro A, Lutolf MP. 3D niche microarrays for systems-level analyses of cell fate. Nat Commun. 2014;5. doi:10.1038/ncomms5324.

84. Di Maggio N, Piccinini E, Jaworski M, Trumpp A, Wendt DJ, Martin I. Toward modeling the bone marrow niche using scaffold-based 3D culture systems. Biomaterials. 2011;32(2): 321-9. doi:10.1016/j.biomaterials.2010.09.041.

85. Seliktar D. Designing cell-compatible hydrogels for biomedical applications. Science. 2012;336(6085):1124-8. doi:10.1126/ science.1214804.

86. Fedorovich NE, Oudshoorn MH, van Geemen D, Hennink WE, Alblas J, Dhert WJA. The effect of photopolymerization on stem cells embedded in hydrogels. Biomaterials. 2009;30(3):344-53. doi:10.1016/j.biomaterials.2008.09.037.

87. Hennink WE, van Nostrum CF. Novel crosslinking methods to design hydrogels. AdvDrug Deliv Rev. 2012;64(Supplement): 223-36. doi:10.1016/j.addr.2012.09.009.

88. Kloxin AM, Tibbitt MW, Anseth KS. Synthesis of photodegradable hydrogels as dynamically tunable cell culture platforms. Nat Protoc. 2010;5(12):1867-87. doi:10.1038/nprot.2010.139.

89. Xing J, Liu L, Song X, Zhao Y, Zhang L, Dong X, et al. 3D hydrogels with high resolution fabricated by two-photon polymerization with sensitive water soluble initiators. J Mater Chem B. 2015;3(43):8486-91. doi:10.1039/c5tb01545f.

90. Lewis KJR, Anseth KS. Hydrogel scaffolds to study cell biology in four dimensions. MRS Bull. 2013;38(03):260-8. doi:10.1557/ mrs.2013.54.

91. Tibbitt MW, Kloxin AM, Sawicki LA, Anseth KS. Mechanical properties and degradation of chain and step-polymerized photodegradable hydrogels. Macromolecules. 2013;46(7):2785-92. doi:10.1021/ma302522x

92. Ferreira MS, Jahnen-Dechent W, Labude N, Bovi M, Hieronymus $\mathrm{T}$, Zenke M, et al. Cord blood-hematopoietic stem cell expansion in 3D fibrin scaffolds with stromal support. Biomaterials. 2012;33(29):6987-97. doi:10.1016/j.biomaterials.2012.06.029.

93. Raic A, Rodling L, Kalbacher H, Lee-Thedieck C. Biomimetic macroporous PEG hydrogels as 3D scaffolds for the multiplication of human hematopoietic stem and progenitor cells. Biomaterials. 2014;35(3):929-40. doi:10.1016/j.biomaterials.2013.10.038.

94. Tan J, Liu T, Hou L, Meng W, Wang Y, Zhi W, et al. Maintenance and expansion of hematopoietic stem/progenitor cells in biomimetic osteoblast niche. Cytotechnology. 2010;62(5):439-48. doi: 10.1007/s10616-010-9297-6.

95. Hutson CB, Nichol JW, Aubin H, Bae H, Yamanlar S, Al-Haque $\mathrm{S}$, et al. Synthesis and characterization of tunable poly(ethylene glycol): gelatin methacrylate composite hydrogels. Tissue Eng A. 2011;17(13-14):1713-23. doi:10.1089/ten.tea.2010.0666.
96. Hudalla GA, Koepsel JT, Murphy WL. Surfaces that sequester serum-borne heparin amplify growth factor activity. Adv Mater. 2011;23(45):5415-8. doi:10.1002/adma.201103046.

97. Belair DG, Le NN, Murphy WL. Design of growth factor sequestering biomaterials. Chem Commun. 2014;50(99):15651-68. doi: 10.1039/c4cc04317k.

98. Hudalla GA, Kouris NA, Koepsel JT, Ogle BM, Murphy WL. Harnessing endogenous growth factor activity modulates stem cell behavior. Integr Biol. 2011;3(8):832-42. doi:10.1039/clib00021g.

99. Lim JJ, Temenoff JS. The effect of desulfation of chondroitin sulfate on interactions with positively charged growth factors and upregulation of cartilaginous markers in encapsulated MSCs. Biomaterials. 2013;34(21):5007-18. doi:10.1016/j. biomaterials.2013.03.037.

100. Sakiyama-Elbert SE, Hubbell JA. Controlled release of nerve growth factor from a heparin-containing fibrin-based cell ingrowth matrix. J Control Release. 2000;69(1):149-58. doi:10. 1016/S0168-3659(00)00296-0.

101. Hortensius RA, Becraft JR, Pack DW, Harley BAC. The effect of glycosaminoglycan content on polyethylenimine-based gene delivery within three-dimensional collagen-GAG scaffolds. Biomater Sci. 2015;3(4):645-54. doi:10.1039/c5bm00033e.

102. Sugimura R. Bioengineering hematopoietic stem cell niche toward regenerative medicine. Adv Drug Deliv Rev. 2015. doi:10.1016/j. addr.2015.10.010.

103. Whisler JA, Chen MB, Kamm RD. Control of perfusable microvascular network morphology using a multiculture microfluidic system. Tissue Eng C Methods. 2014;20(7):543-52. doi:10. 1089/ten.TEC.2013.0370.

104. Moya ML, George SC. Integrating organ-specific function with the microcirculation. Curr Opin Chem Eng. 2014;3:103-11. doi: 10.1016/j.coche.2013.12.004.

105. Mahadik BP, Wheeler TD, Skertich LJ, Kenis PJ, Harley BA. Microfluidic generation of gradient hydrogels to modulate hematopoietic stem cell culture environment. Adv Healthcare Mater. 2014;3(3):449-58. doi:10.1002/adhm.201300263.

106. Lecault V, Vaninsberghe M, Sekulovic S, Knapp DJ, Wohrer S, Bowden W, et al. High-throughput analysis of single hematopoietic stem cell proliferation in microfluidic cell culture arrays. Nat Methods. 2011;8(7):581-6. doi:10.1038/nmeth.1614.

107. Schroeder T. Hematopoietic stem cell heterogeneity: subtypes not unpredictable behavior. Cell Stem Cell. 2010;6(3):203-7. doi:10. 1016/j.stem.2010.02.006.

108. Perry JM, Li L. Functional assays for hematopoietic stem cell selfrenewal. In: Ding S, editor. Cellular programming and reprogramming: methods and protocols. Methods Mol Biol. 2010:45-54.

109. Frisz JF, Choi JS, Wilson RL, Harley BAC, Kraft ML. Identifying differentiation stage of individual primary hematopoietic cells from mouse bone marrow by multivariate analysis of TOFsecondary ion mass spectrometry data. Anal Chem. 2012;84(10): 4307-13. doi:10.1021/ac203329j.

110. Ilin Y, Kraft ML. Secondary ion mass spectrometry and Raman spectroscopy for tissue engineering applications. Curr Opin Biotechnol. 2015;31:108-16. doi:10.1016/j.copbio.2014.10.011.

111. Chen W, Long KD, Lu M, Chaudhery V, Yu H, Choi JS, et al. Photonic crystal enhanced microscopy for imaging of live cell adhesion. Analyst. 2013;138(20):5886-94. doi:10.1039/c3an01541f.

112. Chen W, Long KD, Yu H, Tan Y, Choi JS, Harley BAC, et al. Enhanced live cell imaging via photonic crystal enhanced fluorescence microscopy. Analyst. 2014;139(22):5954-63. doi:10.1039/ C4AN01508H.

113. Chen W, Long KD, Kurniawan J, Hung M, Yu H, Harley BA, et al. Planar photonic crystal biosensor for quantitative label-free cell attachment microscopy. Adv Optic Mater. 2015;3(11):1623-32. doi:10.1002/adom.201500260. 